\title{
A load frame for in situ tomography at PETRA III
}

\author{
Julian Moosmann**a $^{*^{\mathrm{a}}}$, D. C. Florian Wieland ${ }^{\mathrm{a}}$, Berit Zeller-Plumhoff ${ }^{\mathrm{a}}$, Silvia Galli ${ }^{\mathrm{b}}$, Diana Krüger ${ }^{\mathrm{a}}$, \\ Alexey Ershov ${ }^{c}$, Silke Lautner ${ }^{\mathrm{d}}$, Julian Sartori ${ }^{\mathrm{e}}$, Mason Dean ${ }^{\mathrm{f}}$, Sebastian Köhring ${ }^{\mathrm{g}}$, Hilmar \\ Burmester $^{\mathrm{a}}$, Thomas Dose ${ }^{\mathrm{a}}$, Niccolò Peruzzi ${ }^{\mathrm{h}}$, Ann Wennerberg ${ }^{\mathrm{i}}$, Regine Willumeit-Römer ${ }^{\mathrm{a}, \mathrm{j}}$, Fabian \\ Wilde $^{\mathrm{a}}$, Philipp Heuser ${ }^{\mathrm{k}}$, Jörg U. Hammel ${ }^{\mathrm{a}}$, Felix Beckmann ${ }^{\mathrm{a}}$, \\ ${ }^{a}$ Institute of Materials Research, Helmholtz-Zentrum Geesthacht, Max-Planck-Str. 1, 21502 \\ Geesthacht, Germany; \\ ${ }^{\mathrm{b}}$ Faculty of Odontology, Malmö University, 20506 Malmö, Sweden; \\ 'Institute for Photon Science and Synchrotron Radiation, Karlsruhe Institute of Technology, \\ Herrmann-von-Helmholtz-Platz 1, 76344 Eggenstein-Leopoldshafen, Germany; \\ ${ }^{\mathrm{d}}$ Faculty of Wood Engineering, Eberswalde University for Sustainable Development, Schicklerstr. 5, \\ 16225 Eberswalde, Germany; \\ ${ }^{\mathrm{e}}$ Institute of Zoology and Evolutionary Research, Friedrich-Schiller-University Jena, Erbertstr. 1, \\ 07743 Jena, Germany; \\ fDepartment of Biomaterials, Max Planck Institute of Colloids and Interfaces, 14424 Potsdam, \\ Germany; \\ ${ }^{g}$ Biomechatronics Group, Faculty of Mechanical Engineering, Technische Universitaet Ilmenau, \\ Max-Planck-Ring 12, 98693 Ilmenau, Germany; \\ ${ }^{\mathrm{h}}$ Clinical Sciences, Lund University, 22185 Lund, Sweden; \\ ${ }^{\mathrm{i}}$ Institute of Odontology, University of Gothenburg, 40530 Gothenburg, Sweden; \\ ${ }^{\mathrm{j}}$ Institute of Materials Science, Christian-Albrechts-Universität zu Kiel, Christian-Albrechts-Platz 4, \\ 24118 Kiel, Germany; \\ ${ }^{k}$ DESY Informationstechnologies, Deutsches Elektronen-Synchrotron DESY, Notkestr. 85, 22607 \\ Hamburg, Germany;
}

\begin{abstract}
A load frame for in situ mechanical testing is developed for the microtomography end stations at the imaging beamline P05 and the high-energy material science beamline P07 of PETRA III at DESY, both operated by the HelmholtzZentrum Geesthacht. The load frame is fully integrated into the beamline control system and can be controlled via a feedback loop. All relevant parameters (load, displacement, temperature, etc.) are continuously logged. It can be operated in compression or tensile mode applying forces of up to $1 \mathrm{kN}$ and is compatible with all contrast modalities available at IBL and HEMS i.e. conventional attenuation contrast, propagation based phase contrast and differential phase contrast using a grating interferometer. The modularity and flexibility of the load frame allows conducting a wide range of experiments. E.g. compression tests to understand the failure mechanisms in biodegradable implants in rat bone or to investigate the mechanics and kinematics of the tessellated cartilage skeleton of sharks and rays, or tensile tests to illuminate the structure-property relationship in poplar tension wood or to visualize the 3D deformation of the tendonbone insertion. We present recent results from the experiments described including machine-learning driven volume segmentation and digital volume correlation of tomography sequences under increasing load conditions.
\end{abstract}

Keywords: X-ray tomography, in situ, synchrotron radiation, load frame, mechanical testing, compression, tensile testing, dose, digital volume correlation, segmentation

*julian.moosmann@hzg.de; phone +49 40 8998-6904; www.hzg.de 


\section{INTRODUCTION}

In order to investigate the relationship between microstructure and mechanics, in situ tomography under load conditions has become a valuable tool. Here, we report on the development of load frames for mechanical testing combined with in situ synchrotron radiation based X-ray imaging at the microtomography end stations at the imaging beamline (IBL) ${ }^{1-3}$ P05 and the high-energy material science (HEMS) ${ }^{4}$ beamline P07 of PETRA III at DESY, both operated by the Helmholtz-Zentrum Geesthacht (HZG). In addition to a visual inspection, the acquired 4D data sets can further be analyzed by means of digital volume correlation in order to compute strain (or displacement) fields. The computed strain fields can then further serve as input for simulations, to validate a simulation against it, or to identify, track and visualize failure mechanisms in the sample. Exemplarily on the research on biodegradable implants, we discuss important aspects of in situ tomography experiments i.e. sample mounting, restrictions by the deposited X-ray dose, and the postprocessing and analysis of the acquired data sets. Further applications using propagation based phase contrast are shown investigating the structure-property relationship in poplar wood and the mechanics and kinematics of the tessellated skeleton of sharks and rays. Also included are tensile tests of murine tendon insertions in which we compared grating interferometric and propagation based phase contrast with a load frame developed and provided by Technische Universität Ilmenau.

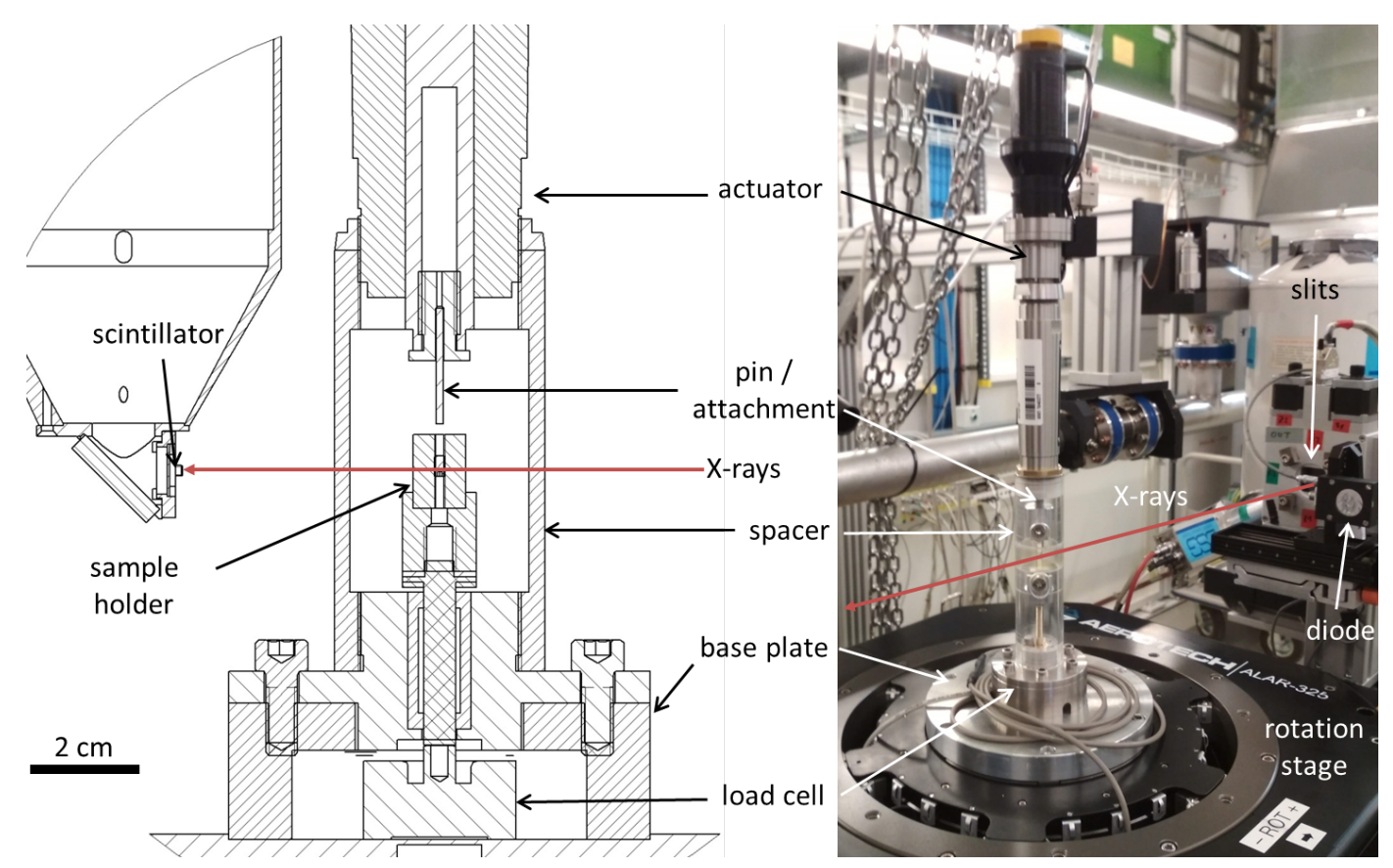

Figure 1: Load frame for in situ tomography under load conditions. Left: Schematic drawing of the load frame. Right: Photograph of the load frame installed on top of the rotation stage at the microtomography end station at the imaging beamline (IBL) at P05 at PETRA III at DESY. The load cell is integrated into the base plate which is attached to the rotation stage. Force closure is accomplished via an X-ray transparent spacer on top of which the actuator is mounted.

\section{LOAD FRAME}

The load frame, which was employed in the experiment presented in sections 3.1, 3.2, and 3.3, was funded by the Röntgen-Ångström (see Acknowledgements) in order to investigate the failure mechanisms in biodegradable implants 
and is freely available for general users at the HZG imaging beamlines. The frame was designed to offer a high flexibility and a high modularity allowing to be used for a wide range of applications and samples. The load frame setup is compatible with all imaging modalities available at the microtomography end stations at IBL and HEMS, i.e. conventional absorption contrast, grating ${ }^{5}$ and propagation based phase contrast ${ }^{6,7}$. In Figure 1 , a technical drawing of the load frame is depicted. The base plate of the frame is mounted on top of the rotation axis and includes the load cell. An $\mathrm{X}$-ray transparent, cylindrical spacer connects the base plate and the actuator and thus provides force closure. Forces are applied to the sample from atop via the actuator and are relayed by a pin to the load cell. The frame can be operated in compression and tension mode. The current actuator (customized Linear-Aktuator M-35, PI miCos GmbH, Eschbach, Deutschland) offers a resolution of $100 \mathrm{~nm}$ per full step with 200 steps per revolution and can exert forces up to $1 \mathrm{kN}$ with a maximum travel range of about $100 \mathrm{~mm}$. The signal from the load cell is transmitted to the signal amplifier (Hottinger Baldwin Messtechnik $\mathrm{GmbH}$ ) via the slip ring of the rotation axis. The signal can be directly read from the beamline control system and is continuously logged at user-defined intervals using a status server. In addition to the signal from the load cell, the status server allows to log other parameters and devices such as actuator displacement, temperature, etc. Various load cells (Burster Präzisionsmesstechnik GmbH \& Co KG) with different maximum nominal forces and accuracies are available. The X-ray transparent spacer is typically made from polyether ether ketone (PEEK). PEEK has excellent mechanical properties, is stable during X-ray irradiation, and is easily machined. The wall thickness and the diameter of the spacer can be adopted for the envisaged forces and other experimental constraints using custom made spacers. For experiments where only low forces are applied, spacers made of acrylic glass can be used. However, acrylic glass becomes brittle when irradiated with X-rays, e.g. see Figure 7a, and must be replaced after 1-3 days of experiment. For sample mounting and/or alignment, 3D printers are available at HZG and DESY in order to produce custom-made sample holders, e.g. see 3.1.1. To apply forces the actuator is moved by displacement or using a feedback control loop to reach a given target force, e.g. see force plots in Figure 2c, Figure 7b or Figure $8 \mathrm{~b}$ where the feedback control loop was used.
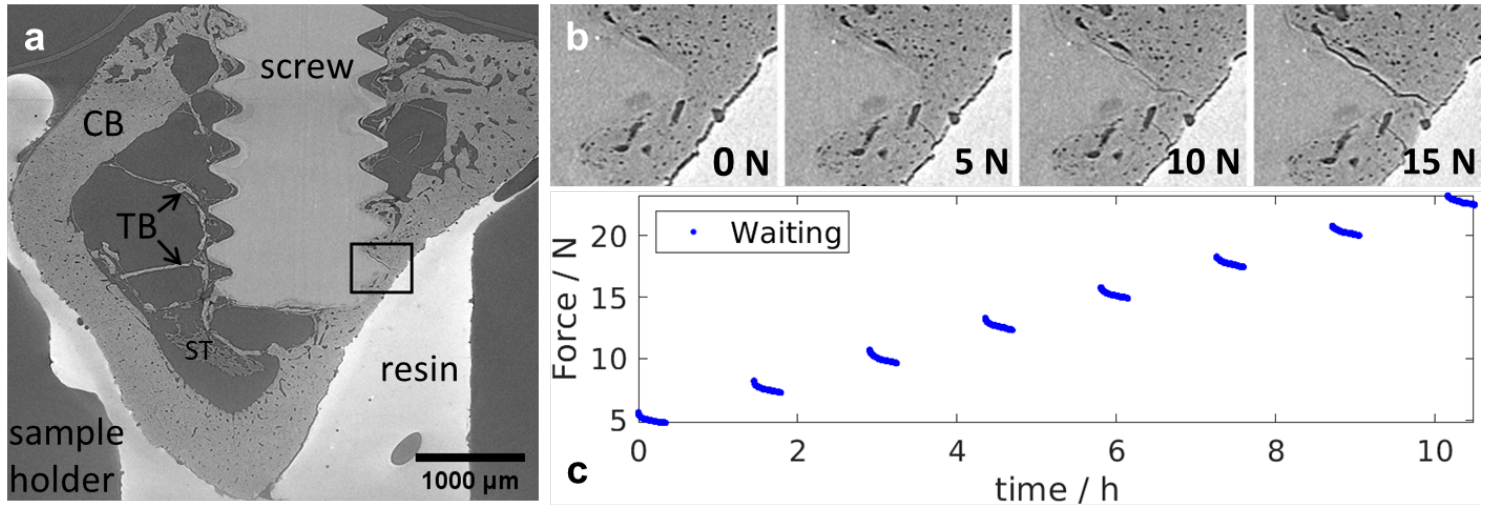

Figure 2: In situ tomography of a biodegradable screw under compressive load illustrating the issue of a bicortical implantation. (a) Vertical slice through a tomogram of an explant after a compressive load of $15 \mathrm{~N}$ was applied. A screw made of Mg10Gd (4 mm high, $2 \mathrm{~mm}$ diameter) was implanted in a rat femur for 4 weeks. A sample of about $6 \mathrm{~mm}$ height and about $5 \mathrm{~mm}$ width was explanted after the rat was sacrificed. A 3D printed sample mount was used to align and stabilize the explant. For further stabilization a resin-based filling was used. In this experiment, a rather X-ray opaque instead of Xray transparent resin was used accidentally. Cortical (CB) and trabecular bone (TB) can clearly be identified as well as soft tissue (ST) components. The black rectangle indicates the region of interest which is depicted in the image sequence in (b). (b) Selection of images from the acquired load sequence. The force was increased from $0 \mathrm{~N}$ to $22.5 \mathrm{~N}$ in steps of $2.5 \mathrm{~N}$ as depicted in (c). During force increase fractures occur and propagate within the sample. Due to the bicortical implantation fractures occur at angle of 45 degree which is the angle expected in a fracture occurring due to the compression of a solid block of material. (c) Force recorded during the waiting time after the target force was reached and before data acquisition started. A waiting time of 20 min was used before each tomogram to account for relaxation processes. Due to technical problems the force during tomographic data acquisition was not logged. 


\section{EXPERIMENTS}

\subsection{Compressive testing with attenuation contrast: failure mechanisms in biodegradable implants}

In recent years, biodegradable materials have become more and more attractive for orthopedic and traumatological applications in order to replace permanent implants made of Titanium (Ti) or its alloys ${ }^{8,9}$. Using materials which degrade under physiological conditions avoids to remove the implant in a follow-up surgery which is necessary in the case of children who are still growing, or when detrimental effects occur due to wear debris or allergic reactions, or if demanded by the patient. Magnesium (Mg) and its alloys have gained much interest in medical applications because of its biocompatibility, biodegradability, and mechanical properties ${ }^{10-12}$. While there are already Mg-based implants available on the market, fundamental knowledge about the complex interactions between implant and bone is missing. Therefore, a most comprehensive characterization of the bone-implant interface is a currently ongoing task at HZG. To characterize the biomechanical properties and to elucidate the failure mechanisms of biodegradable Mg-based implants, in situ tomography under compressive load conditions was conducted on explants, e.g. see Figure 2 and Figure 3d. Screws, made of an alloy of $\mathrm{Mg}$ and Gadolinium $(\mathrm{Gd})$, were implanted in the femurs of rat for 4 to 12 weeks.
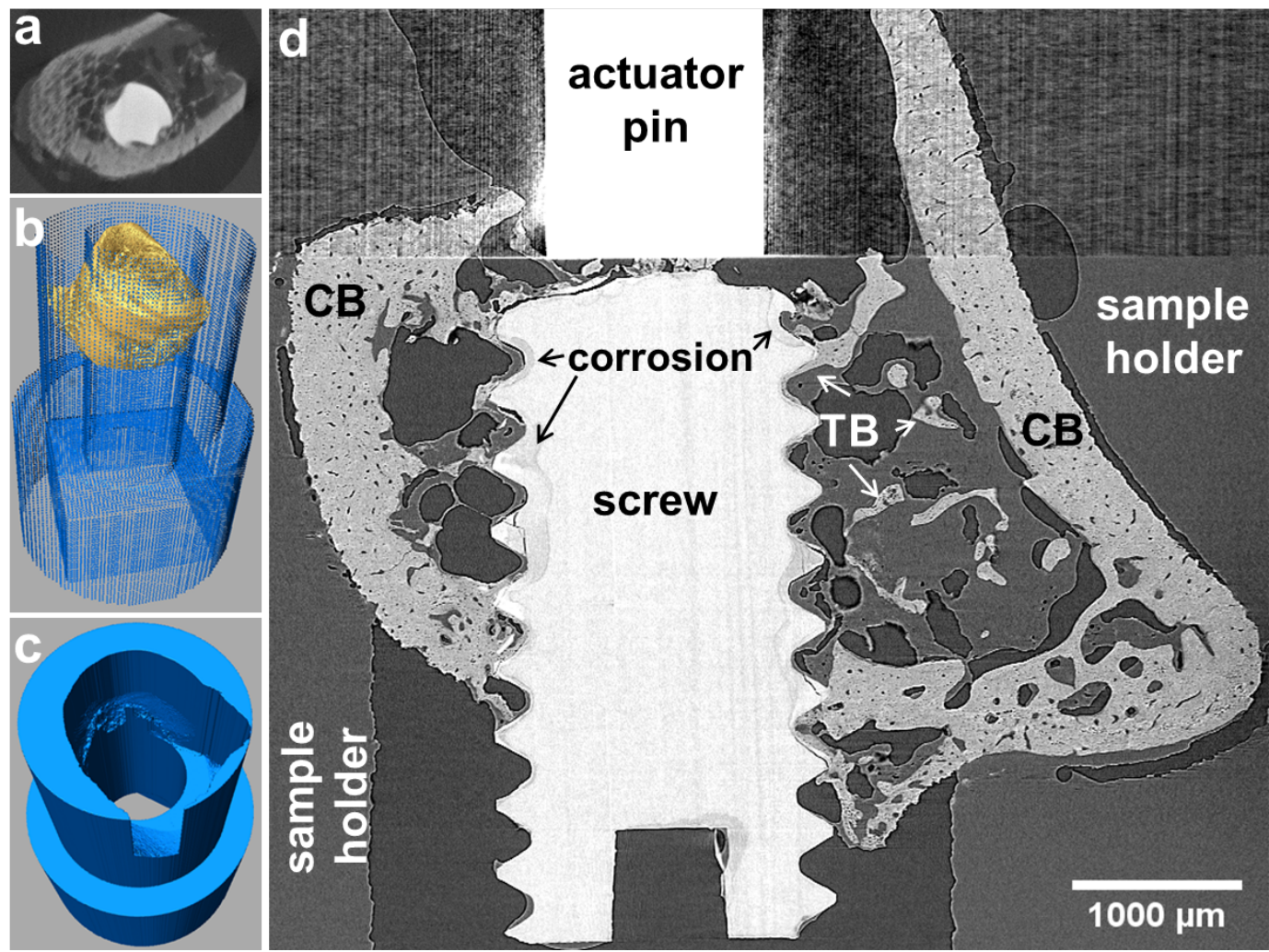

Figure 3: Customized 3D printed sample mount. (a) Horizontal slice through a tomogram which was quickly taken of a frozen sample using a laboratory CT scanner at low spatial resolution and with low image quality. (b) The volume reconstructed from the CT scan in (a) is digitally aligned such that the principal axis of the screw is paraxial to the vertical axis and centered horizontally with the origin. (c) The digitally aligned volume is now used to 3D print a sample holder, the cavity of which is the negative of the explant. Not depicted are additional tubes in the printed holder which are used to insert the filling which further stabilizes the explant in the holder. (d) Vertical slice through a tomogram of an Mg10Gd screw which was implanted in a rat femur for 4 weeks. A two-part sample holder design was used which encloses the sample and which allows to remove the cortical bone (CB) in the region above the bottom of the screw. Applying force onto the bottom of the screw, the implant is pushed out. Thereby, the bone-implant interface is tested instead of the cortical bone as is the case in Figure 2. (The composite filling cannot be distinguished from the printed sample holder in the depicted slice. The artefacts and the minor image quality at the top part of the images are due the full attenuation of X-rays by the actuator pin.) 


\subsubsection{Sample mount}

For the compression experiments on biodegradable implants, individually designed customized sample holders were made using a 3D printer. Using a laboratory $\mathrm{CT}$ scanner (phoenix nanotom s, General Electric) a fast ( $\sim 5 \mathrm{~min})$ tomogram of the frozen sample is acquired. The reconstructed volume is then digitally aligned such that the principal axis of the screw is paraxial to the vertical axis and centered horizontally with the origin. The digitally aligned volume is then used to 3D print a customized sample holder, see Figure 3. This procedure is necessary for several reasons: 1) To stabilize the sample during compression and data acquisition. 2) To align the principal axis of the implanted screw paraxially to the force applied by the actuator. To further increase stability, a resin-based composite filling is used which can be inserted through channels in the sample holder between the outer surface of the cortical bone and inner surface of the sample holder. The composite (easyform LC, DETAX GmbH \& Co. KG, Ettlingen, Germany) is a light-curing, X-ray transparent resin gel which is dimensionally stable and cured with blue light within a few seconds. 3) To center the sample within the field of view i.e. to align the principal axis of the screw with the rotation axis. 4) To assure a proper mechanical testing of the bone-implant interface, a special sample treatment is necessary. This is due to the fact that, even though the surgeon tried to implant the screws monocortically in the rat bone, the bottom end of the screw is in contact with the cortical bone opposite to the implantation site in almost all samples. Pushing onto such an implanted screw is rather a test of the stability of the cortical bone with which the implant is in contact, instead of the bone-implant interface, see Figure 2a. As expected from material science considerations, ruptures within the cortical bone occur at 45 degree angle, see Figure $2 \mathrm{~b}$. To mechanically test the bone-implant interface rather than the cortical bone, a further refinement of the sample holder as depicted in Figure 2a is required. A two-part sample holder is printed that completely encloses the explant, see Figure 3d. The cavity in the top of the 3D printed holder is now used as guidance rail to remove the cortical bone with a dental drill (before in situ tomography). Forces can now be applied to the bottom of the screw through the cortical bone opposite to the implantation site, and the implant can now be pushed out of the bone (into the bottom cavity of the sample holder).
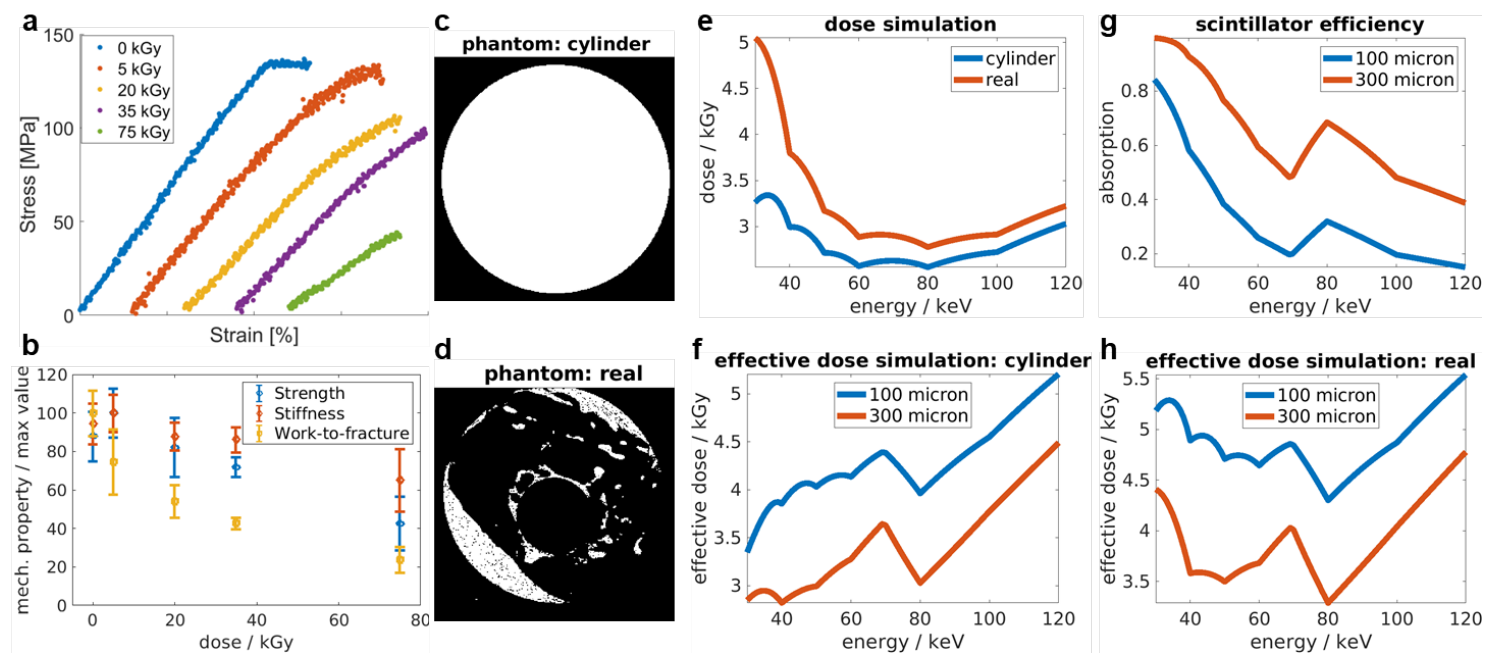

Figure 4: Effect of irradiation dose on the mechanical properties of hydrated rat bone $(a, b)$ and simulation of the dose deposited in bone (d-h). (a) Stress-strain curves of rectangular samples of rat femurs which were exposed to increasing levels of irradiation: $0 \mathrm{kGy}, 5 \mathrm{kGy}, 20 \mathrm{kGy}, 35 \mathrm{kGy}, 75 \mathrm{kGy}$. Graphs are shifted on the x-axis for clarity. (b) Effects of irradiation on ultimate bending strength, bending stiffness and work-to-fracture. The plotted results are normalized to the highest value for each group; Error bars represent the standard deviation. The progressive degradation of mechanical properties with increasing doses of irradiation is in agreement with the observation by Bart et al. on human cortical bone $\mathrm{e}^{13,14}$. (c,d) Horizontal cross-sections of phantoms used for the dose simulations in (e-h): (c) homogeneous cylinder with a diameter of $7 \mathrm{~mm}$, (d) phantom generated from the segmented bone structure of the measurement of a critically-point dried explant of a Mg10Gd screw implanted in a rat femur for 4 weeks. (e) Dose-energy relation calculated for the cylindrical (c) and realistic (d) phantom. (g) Efficiency of a scintillator made of cadmium tungstate $\left(\mathrm{CdWO}_{4}\right)$ for two different scintillator thicknesses. Values are normalized to the highest value of both curves within the considered energy range. (e,f) Effective, i.e. scintillator efficiency scaled, dose plots for the both phantoms. The graphs depict the dose, plotted in (b), multiplied by a scaling factor derived from the scintillator efficiency depicted in (g). 


\subsubsection{Dose}

When conducting mechanical tests in combination with in situ tomography special attention has to be taken regarding the effects of the X-ray dose that is deposited in the sample during the experiment. Barth et al. have examined the effects of irradiation dose on the mechanical aspects of deformation and fracture in human cortical bone ${ }^{13,14}$. At about $35 \mathrm{kGy}$ of irradiation dose, macroscopic properties of bone such as bone strength, ductility and fracture resistance start to progressively degrade with increasing levels of irradiation. At about $70 \mathrm{kGy}$ of irradiation, bone plasticity is essentially suppressed. A typical high quality tomogram acquired at synchrotron radiation facility at about $1 \mu \mathrm{m}$ effective pixel size can easily reach $1 \mathrm{MGy}$ of irradiation dose. Thus, data acquisition has to be optimized to reduce the dose acquired during the load sequence.

Mechanical tests on rectangular samples of femoral rat bone, which have been exposed to different dose levels, have been conducted in order to relate the critical radiation threshold in humans to rats. The samples have been irradiated immersed in HBSS (Hank's balanced salt solution) to avoid dehydration. For the mechanical tests, the dose was estimated analytically as in Barth et al. ${ }^{13}$. Figure 4a depicts stress-strain curves of rectangular samples of rat femurs which were exposed to increasing levels of irradiation ( $0 \mathrm{kGy}, 5 \mathrm{kGy}, 20 \mathrm{kGy}, 35 \mathrm{kGy}, 75 \mathrm{kGy})$. Three-point bend tests were performed on three samples per irradiation level using a support span of $12 \mathrm{~mm}$, an applied force diameter of $2 \mathrm{~mm}$ and a displacement rate of $10 \mu \mathrm{m} / \mathrm{s}$. The depicted curves show a similar behavior as in Barth et al. ${ }^{13,14}$. Graphs are shifted horizontally for clarity. From the resulting stress-strain curves, following parameters were determined: the ultimate bending strength and strain at the point of maximum load, the bending stiffness which is proportional to the slope of the linear part of the stress-strain curves, and the work-to-fracture which is the integral of the stress-strain curve from zero strain until the point of fracture. The parameters depicted in Figure $4 \mathrm{a}$ are normalized by the maximum value within each group. While the ultimate bending strain did not reveal significant variations between the different irradiation levels, all the mechanical properties depicted in Figure $4 \mathrm{~b}$ exhibit a progressive degradation with the increasing levels of irradiation dose which is in agreement with the observation by Bart et al. on human cortical bone ${ }^{13,14}$. The results of this preliminary analysis thus corroborate to assume the critical dose level to be similar in rats and humans.

To choose an optimal energy, a simulation of the dose that is deposited in the sample at different scan parameters was conducted. The energies considered range from $30 \mathrm{keV}$ to $120 \mathrm{keV}$ (in $1 \mathrm{keV}$ steps). Two phantoms with a mass attenuation coefficient of cortical bone with different geometries were considered. First, a homogeneously filled cylinder and second, a more realistic phantom generated from a tomogram of an explant where the bone structure was extracted by segmentation, see Figure $4 \mathrm{c}$ and d, respectively. Volumes were downsampled four-fold to speed up the simulation. 1200 projections with $50 \mathrm{~ms}$ exposure times were used. For simplicity, the mass attenuation coefficient of cortical bone was also assumed for the trabecular structures in the realistic phantom. Tabulated values of the mass attenuation coefficients were taken from the database of the national institute of standards and technology (NIST) ${ }^{15}$. Using the forward projector of the ASTRA toolbox, the absorbed energy was calculated for each projection assuming BeerLamberts law. The mass of the phantoms was calculated from the segmented volumes assuming a density of cortical bone of $1920 \mathrm{~kg} / \mathrm{m}^{3}$. The dose is then given by the absorbed energy per tomogram divided by the mass.

For the dose simulations depicted in Figure 4e-h, the flux was fixed at the value which was measured with a diode at an energy of $45 \mathrm{keV}$ at IBL. The photon flux at IBL was reduced by an absorber (aluminum alloy) to be compatible with the scan parameters such that an estimated dose of about $3.5 \mathrm{kGy}$ per tomogram was achieved. Absorption by the PEEK spacer of the load frame was accounted for (density: $1320 \mathrm{~kg} / \mathrm{m}^{3}$ ). As expected by the exponential attenuation of the Beer-Lambert law, the dose in the homogeneous sample is smaller than in the realistic sample of similar size, but with a much finer bone structure. Both plots exhibit local and global minima at about $60 \mathrm{keV}$ and $80 \mathrm{keV}$, respectively, and increase towards lower energies due to the increased attenuation (or imaginary part of the refractive index) and towards higher energies due to the higher energy of photons interacting with the sample. Water and soft tissue surrounding the bone structure as well as the sample holder were not yet included in the simulation. Thus, the actual dose will be lower than the calculated dose and the calculated values can be considered as upper limit. The simulation in Figure $4 \mathrm{e}$, however, does not account for increased scan times resulting from the lower scintillator efficiency at higher energies as plotted in Figure 4g for a scintillator thickness of $100 \mu \mathrm{m}$ and $300 \mu \mathrm{m}$. To account for the scintillator efficiency, an effective dose was calculated where the calculated dose as plotted in Figure 4e was multiplied by a scaling factor which is an affine function of the scintillator transmission. The scaling factor is 1 at $45 \mathrm{keV}$ and for the $300 \mu \mathrm{m}$ thick scintillator. These are the parameters used for the load sequences acquired at IBL and where the photon flux was measured. A considerably different behavior is observed for the effective dose as depicted in Figure $4 \mathrm{f}$ and $\mathrm{h}$ compared to the simulation not accounting for the scintillator efficiency as in Figure 4e. There is a distinct minimum at about 80 
$\mathrm{keV}$. A local minimum is observed at about $50 \mathrm{keV}$ which is the highest possible energy at IBL. For the load experiments on biodegradable implants at IBL, a slightly lower energy of $45 \mathrm{keV}$ was chosen. Not accounted for in this simulation is the reduction of the signal to noise ratio due the decrease of the real part decrement and the imaginary part of the refractive index with increasing energy (apart from absorption edges).
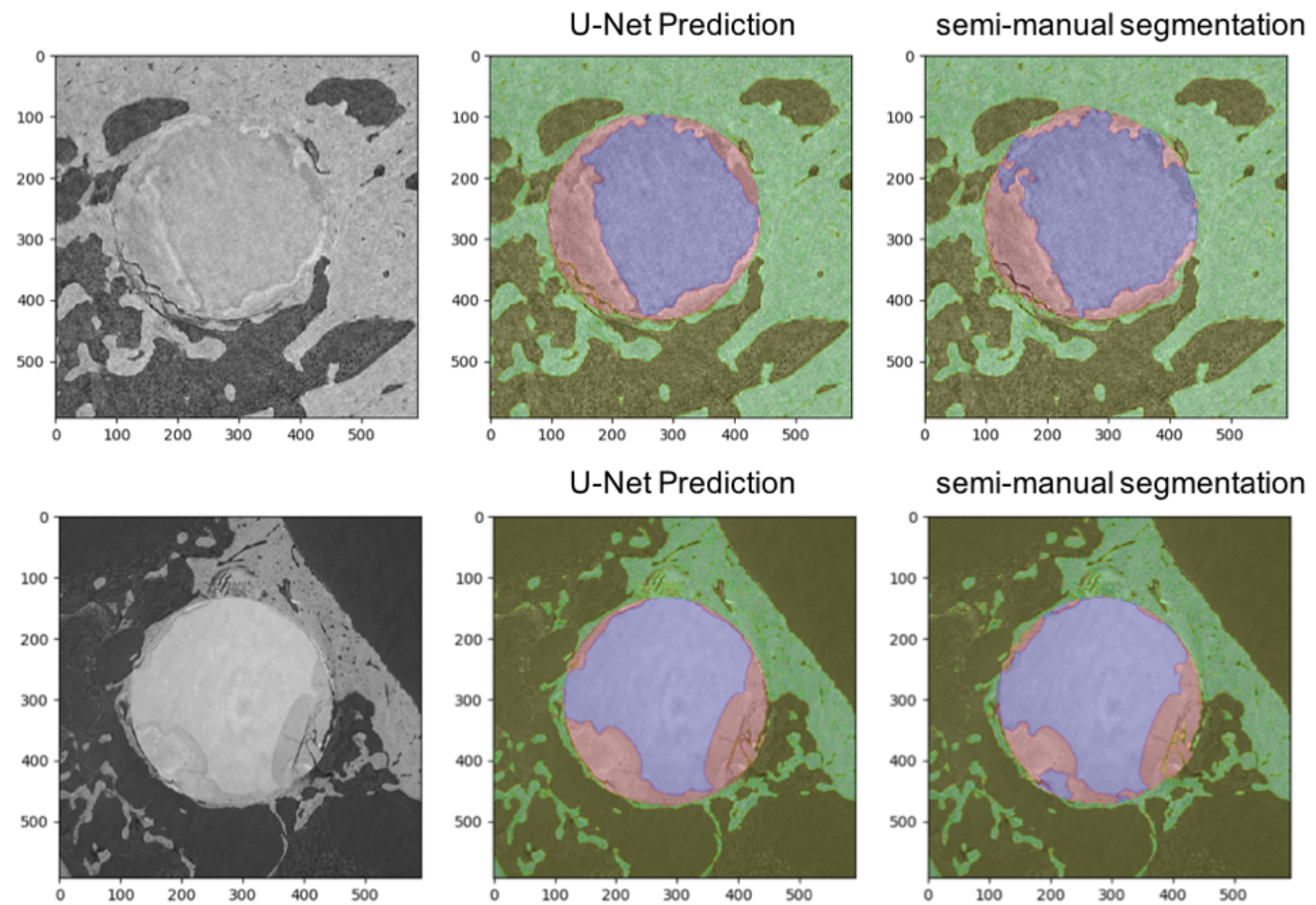

Figure 5: Comparison of the segmentation by the trained U-Net with the semi-manually obtained segmentation. (blue: residual screw, red: corrosion, green: bone). Although trained on weakly annotated data (incomplete, inconsistent, partially false labels), the U-Net Prediction outperforms the semi-manual segmentation. A quantitative evaluation of the results is work in progress. Data sets were resampled to an effective pixel size of $5 \mu \mathrm{m}$.

\subsubsection{Segmentation}

For the quantitative and qualitative evaluation of the imaged 3D volumes, an automatic segmentation procedure, yielding precise voxel-wise labels is essential. Numerous publications ${ }^{16-19}$ indicate that the utilization of artificial intelligence, namely convolutional neural networks $(\mathrm{CNN})$, is well suited for semantic segmentation of biomedical (3D) images. We have trained a U-Net ${ }^{20}$ with five convolutional layers with 64 to 1024 kernels and five deconvolutional layers. A rectified linear units $(\mathrm{ReLu})$ has been used as activation function for all layers, except the last one where softmax is applied. The training has been done with Adam optimizer ${ }^{21}$ and categorical cross-entropy as loss function. We used 2D slices from 12 datasets of $\mathrm{Mg} 5 \mathrm{Gd}$ and $\mathrm{Mg} 10 \mathrm{Gd}$ screws in rat bones with corresponding semi-manually obtained annotations (iterative watershed segmentation with manual adjustments). The training-data has been enriched by augmentations ${ }^{22}$ and $2 \mathrm{D}$ slices from the data were obtained from the three orthogonal directions of the volume. In total 95652 2D images were used for the training. CNN training was implemented in $\mathrm{Keras}^{23}$ and run on single Nvidia V100 GPU nodes of the high performance computing cluster (Maxwell) at DESY. The trained network is used to run inferences from the three orthogonal view axes of the volume and the final label gets assigned based on the label getting the highest probability in one of these three independent segmentations. These triple segmentations mostly remove false classified artefacts which occasionally occur when the segmentation is only using 2D slices from one direction. The validation accuracy at the end of the training is $97 \%$. However, visual inspection of the automated segmentation clearly shows that the results obtained, significantly outperform the previously used semi-automatic segmentation procedure in terms of accuracy, see Figure 5. 
The automatic segmentation of a 3D volume with $1200 \times 1200 \times 1000$ pixels takes 30 min using a P100 GPU. So far we have applied the trained U-Net to more than 100 tomographic datasets of biodegradable screws. The amount of time for that task using the semi-manual procedure would have been in the order of a few years.
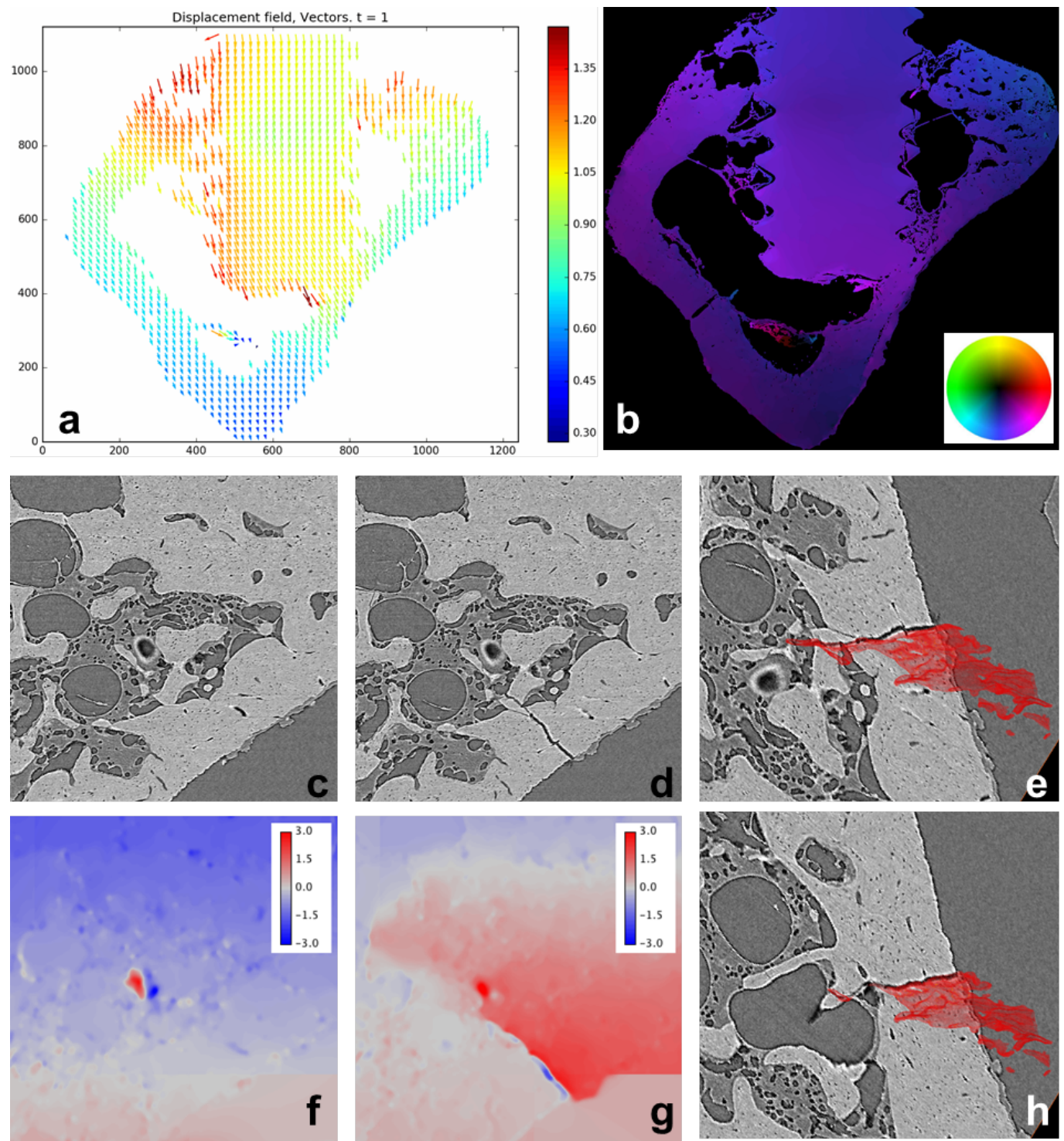

Figure 6: Digital volume correlation via optical flow. (a) The displacement field calculated from two consecutive tomograms at different load is visualized using vectors on a coarse-grained grid projected on a vertical plane. The color bar indicates the displacement in units of voxels. (b) Visualization of the flow field using a color map (bottom right) indicating the direction of the vector field within the depicted plane at voxel resolution. (c,d) Zoomed in region before (c) and after (d) a fracture has occurred. (f,d) The in-plane component of the flow field related to (c) and (d) is depicted. The color bar indicates the amplitude of the in-plane component in units of voxels. (e,h) Rendering of the divergence of the flow field before two different background slices. This illustrates the spatial coincidence of the divergence of the flow field with the occurrence of fractures and demonstrates that the divergence can be used to identify fractures and to track the propagation of which throughout the 4D volumes. 


\subsubsection{Digital volume correlation}

A load tomography sequence of a Mg10Gd screw implanted for 4 weeks in rat femur was used for digital volume correlation via optical flow ${ }^{24-27}$. Optical flow techniques are variational methods where the unknown displacement field is determined as a minimal solution of the energy functional. The energy functional is typically comprised of a data term which assumes constancy of specific image features and a smoothness term which regularizes the spatial variation of the flow field. The displacement (or flow) field will be used for several tasks: 1) To visualize how strain develops during compression and finally failure of the implant as illustrated in Figure 6a and b. In Figure 6a, the flow field is visualized using coarse-grained vectors projected on a vertical plane. In Figure $6 \mathrm{~b}$ a color map is used to indicate the direction of the field vectors at each voxel instead of a vector projection. 2) To identify fractures, to visualize how cracks propagate throughout the 4D volume, and to track fractures back to their origin. Figure $6 \mathrm{c}$ and d depict a region before and after a fracture has occurred. The in-plane component of the corresponding flow field is depicted in Figure $6 \mathrm{f}$ and $\mathrm{g}$. While the vectors, the components, or the magnitude of the flow field can be clearly visualized in a single plane, a 3D presentation of which throughout the full volume becomes messy. For example, trying to identify the crack depicted in Figure $6 \mathrm{~d}$ within the 3D volume using a component of the field as in Figure $6 \mathrm{~g}$ or its magnitude is difficult as the crack becomes indistinguishable from its surrounding when the full volume is rendered. Only when zooming into the yet unknown region of interest the crack becomes clearly visible. Therefore, the divergence of the flow field can be used as demonstrated in Figure 6e and h. Here, the divergence is rendered above two different background slices. This clearly shows that the rendered divergence spatially coincides with the region where a fracture has occurred. 3) Since the Young modulus is unknown throughout the sample, the computed strain field can be used as input in finite element simulations in order to determine the Young modulus. Having determined the Young modulus, the local forces throughout the 4D volume can be computed. Alternatively, the experimentally obtained strain field can be compared with the results of a full-fledged simulation of the implant-bone mechanics.

\subsection{Tensile testing with propagation based phase contrast: structure-property relationship in poplar wood}

In hardwood tree species, tension wood is formed in zones of tension (e.g. branches), which is characterized by a special wall-layer in the fibers, the so-called gelatinous layer (G-layer), generating greater tensile strength at the respective $\operatorname{area}^{28}$. To study the mechanical properties of homogenous samples of tension wood in comparison to normal wood, micro-sections of both types, taken from poplar (Populus nigra), are imaged under tensile load condition until failure using single-distance propagation based phase contrast tomography. A 12-bit CMOS camera with $5120 \times 3840$ pixels and a linear pixel size of $6.4 \mu \mathrm{m}$ was used with $5 \mathrm{x}$ magnification resulting in a field of view of $6.5 \mathrm{~mm} \times 4.9 \mathrm{~mm}$ and an effective pixel size of $1.28 \mu \mathrm{m}$. The scintillator converting X-rays to optical light was made of cadmium tungstate $\left(\mathrm{CdWO}_{4}\right)$. After filtering of faulty pixels, the images were binned $2 \times 2$, before phase retrieval and tomographic reconstruction. 1200 projections and 100 reference image with an exposure time of $100 \mathrm{~ms}$ were taken. Images were acquired at an X-ray energy of $31 \mathrm{keV}$ and a sample to detector distance, $z$, of $0.61 \mathrm{~m}$. All projections were correlated with all reference images using the structural similarity index (SSIM)) to find a best match for the flat-field correction. The correlation is necessary because of temporal instabilities of the X-ray beam. Phase maps were retrieved from flatand dark-field corrected projections using a linearized-in- $z$ transport of intensity equation (TIE). For tomographic reconstruction, filtered backprojection (FBP) was used employing the ASTRA toolbox for the backprojection ${ }^{29,30}$. Data pre-processing, phase retrieval and tomographic reconstruction was done using the tomographic reconstruction pipeline at IBL $^{31,32}$ which is implemented in MATLAB. The image in Figure 7a depicts a poplar wood sample mounted in the load frame after tensile testing. The sample broke during data acquisition after a force of $35 \mathrm{~N}$ was applied, see force plot in Figure 7b. 

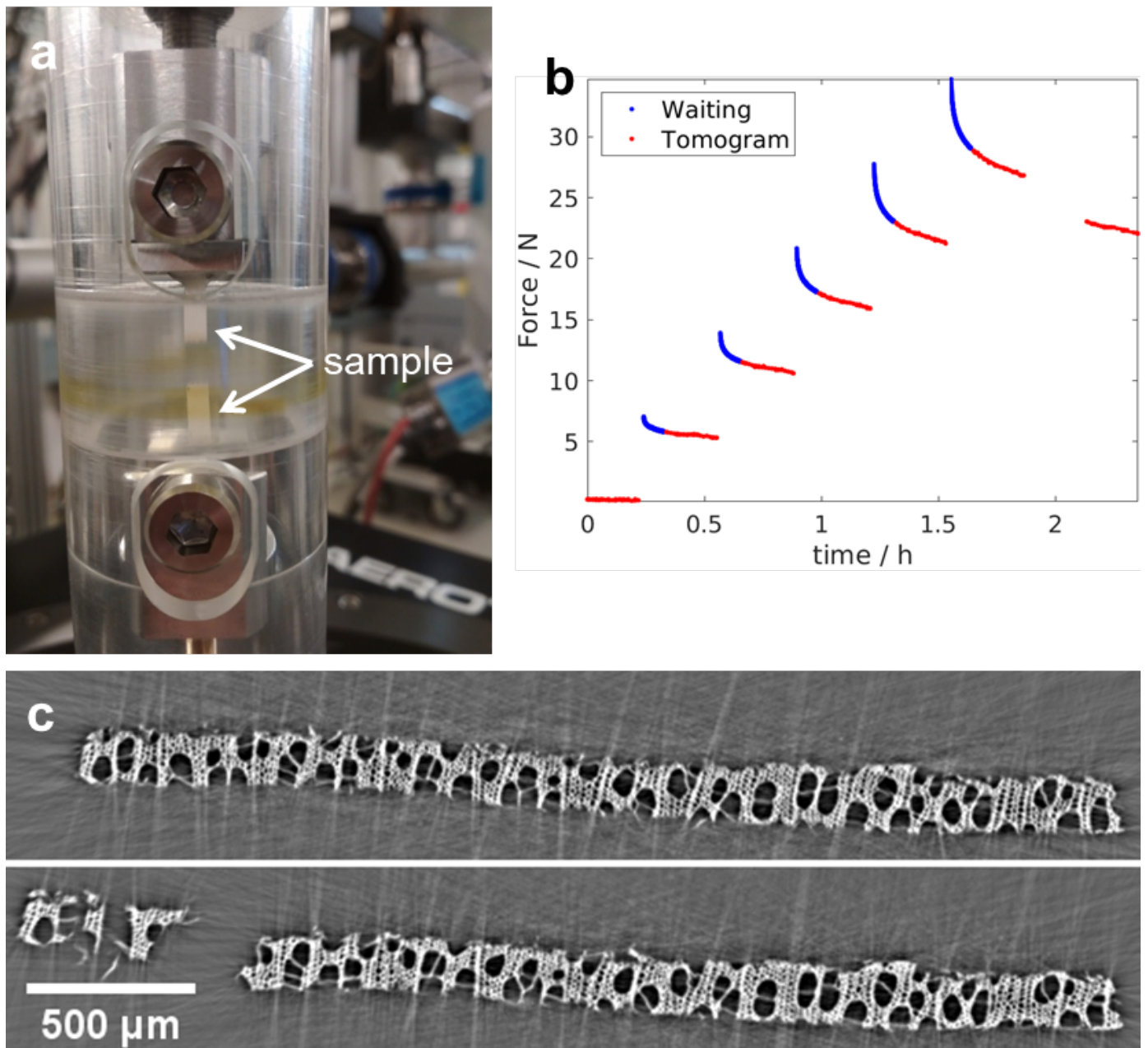

Figure 7: Tensile testing of tension wood. (a) Poplar wood sample mounted in the load frame for tensile testing. The brownish color of the acrylic glass spacer is due to radiation. (b) Force plot during waiting and data tomographic acquisition. After the target force was reached at each load step, a waiting time of 6 min was taken to allow for relaxation processes to take place and to reduce sample movement during data acquisition. (c) Horizontal cross-cuts through tomograms acquired before and after the sample broke.

\subsection{Compressive testing with propagation based phase contrast: mechanics and kinematics of the tessellated skeleton of sharks and rays}

The cartilage skeletons of sharks and rays are covered in mineralized, polygonal tiles (tesserae), which may afford the skeleton flexibility under some loads and stiffness under others ${ }^{33,34}$. Tomographic data sets were acquired under compressive loading in hydrated conditions to provide insights into the tessellated cartilage mechanics and biocomposite structure-function relationships. For the experiment, propagation based phase contrast was used with a sample-to-detector distance of $0.5 \mathrm{~m}$ and an X-ray energy of $35 \mathrm{keV}$. 2400 projections were acquired using a CMOS camera with an effective pixel size of $2.56 \mu \mathrm{m}$ after $2 \times 2$ binning. Phase retrieval assumed the linearized transport of intensity equation (TIE). Data processing as in Section 3.2. A sequence of images under increasing compression where fractures occur is depicted in Figure 8a. The force recorded during the compression sequence is plotted in Figure 8b. 


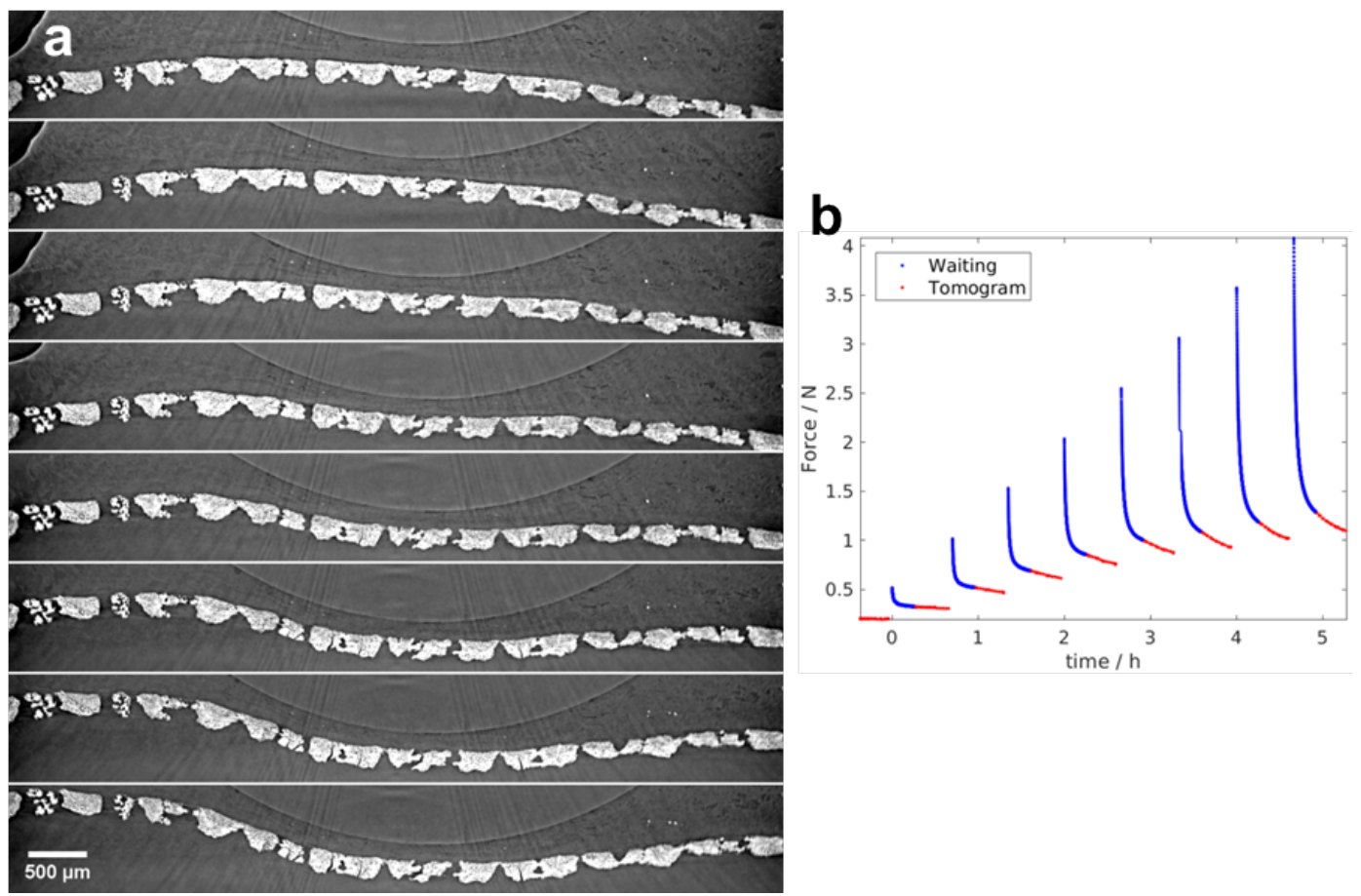

Figure 8: Compression testing of the tessellated skeleton of a shark. (a) Montage of a load sequence with increasing force from top to bottom. (b) Force measured during the load sequence.

\subsection{Tensile testing with propagation based and grating interferometric phase contrast: mouse tendon}

Due to the increased contrast compared to conventional attenuation-contrast imaging, phase contrast imaging techniques have proven to be a valuable tool when investigating weakly absorbing materials like soft tissue ${ }^{35}$. But tendon-bone insertions comprise weakly and strongly attenuating materials. The aim of the experiment by the users from Friedrich Schiller University Jena was to visualize the 3D deformation of the Achilles tendon insertion to the Calcaneus in mice in a tensile test. A customized load frame, as depicted in Figure 9a, was provided by Technische Universität Ilmenau. Single grating interferometry at $23 \mathrm{keV}$ and propagation based phase contrast at $35 \mathrm{keV}$ were tested. Grating-based phase contrast provides a higher dynamic contrast range. The microtomography end stations at the beamlines P05 and P07 at PETRA III at DESY are equipped with a customized set of mechanics to maximize the performance of the interferometer ${ }^{36,37}$. The system allows for phase-contrast measurements in a continuous X-ray energy range between 10 $\mathrm{keV}$ and $80 \mathrm{keV}$. Depending on the material and energy, the setup can achieve a spatial resolution of $5 \mu \mathrm{m}$ on a field of view of $6.5 \mathrm{~mm}$. The fiber diameters of collagen fibers range around $10 \mu \mathrm{m}$ in the examined samples. Propagation based phase contrast was shown to render tissue structure in more detail, see Figure $9 \mathrm{~b}$. 

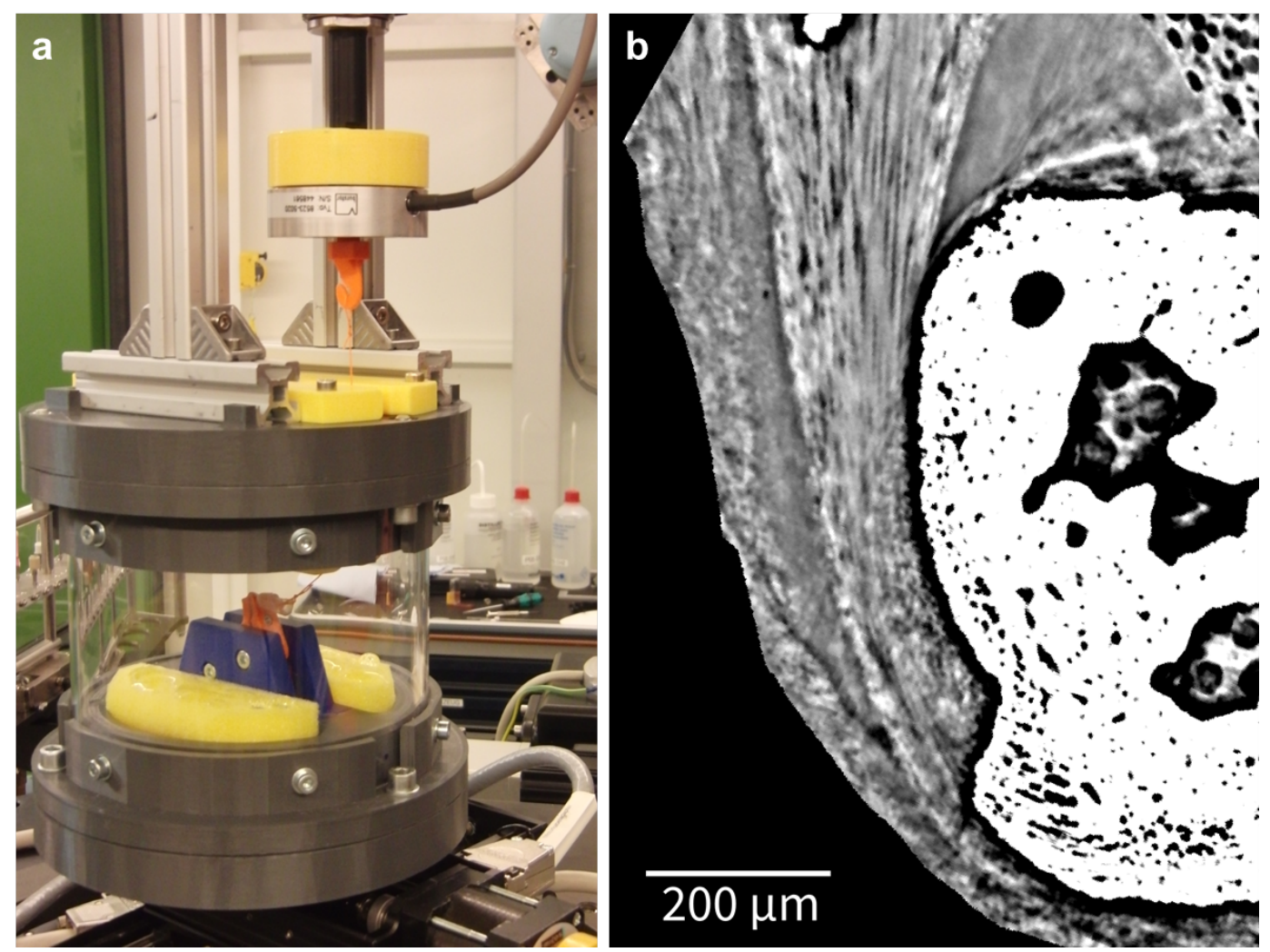

Figure 9: (a) Tensile load frame developed by Technische Universität Ilmenau. (b) Section through a volume data set of a tendon-bone insertion at a tensile load of $0.3 \mathrm{~N}$ acquired with propagation based phase contrast.

\section{SUMMARY AND OUTLOOK}

A load frame for mechanical testing in combination with in situ synchrotron radiation microtomography has been successfully developed for the microtomography end stations at the P05 and P07 beamline at PETRA III at DESY. Experiments under tensile and compressive load conditions in combination with attenuation and (propagation as well as grating interferometric) phase-contrast have been presented. Typical issues that arise in such experiments have been discussed including dose consideration, sample mount, and data analysis.

Modifications of the load frame are in progress to provide a rotary feed through for electric signals and gases or liquids in order to avoid mechanical pull by the piping or cabling and to allow endless rotation. A smaller actuator (with a lower maximum force) will be purchased which can be embedded with a load cell in the cavity within the rotation axis and connected via the slip ring. This will allow for a more compact design and offers better opportunities for tensile tests where there the sample mount typically is a major issue during the design phase and the actual experiment. Further adaptations which allow the application of shear forces are planned.

Regarding the mechanical characterization of the bone-implant interface, HZG is currently working on finite element simulations of the bone mechanics where the displacement fields of the acquired load sequences serve as input in order to determine the Young modulus throughout the sample. Having obtained Young modulus, the actual forces within the sample can be computed giving insight into the failure mechanisms in biodegradable implants. 


\section{ACKNOWLEDGMENTS}

Parts of this research was carried out within the SynchroLoad project (BMBF project number 05K16CGA) and the $\mathrm{MgBone}$ project (BMBF project number $05 \mathrm{~K} 16 \mathrm{CGB}$ ) which are funded by the Röntgen-Ångström Cluster (RÅC), a bilateral research collaboration of the Swedish government and the German Federal Ministry of Education and Research (BMBF). We acknowledge provision of beamtime, related to the proposals I-20160104, I-20160658, II-20170009, I20170074, I-20170402, I-20160046, I-20171047, and I-20170835 at beamlines P05 and P07 at PETRA III at DESY, a member of the Helmholtz Association (HGF). The development of the automatic segmentation is supported by the Helmholtz Association Initiative and Networking Fund under project number ZT-I-0003.

\section{REFERENCES}

[1] Haibel, A., Ogurreck, M., Beckmann, F., Dose, T., Wilde, F., Herzen, J., Müller, M., Schreyer, A., Nazmov, V., Simon, M., Last, A. and Mohr, J., "Micro-and nano-tomography at the GKSS Imaging Beamline at PETRA III," Developments in X-Ray Tomography VII 7804, 78040B, International Society for Optics and Photonics (2010).

[2] Haibel, A., Beckmann, F., Dose, T., Herzen, J., Ogurreck, M., Müller, M. and Schreyer, A., "Latest developments in microtomography and nanotomography at PETRA III," Powder Diffraction 25(2), 161-164 (2010).

[3] Wilde, F., Ogurreck, M., Greving, I., Hammel, J. U., Beckmann, F., Hipp, A., Lottermoser, L., Khokhriakov, I., Lytaev, P., Dose, T., Burmester, H., Müller, M. and Schreyer, A., "Micro-CT at the imaging beamline P05 at PETRA III,” AIP Conference Proceedings 1741(1), 030035 (2016).

[4] Schell, N., King, A., Beckmann, F., Ruhnau, H., Kirchhof, R., Kiehn, R., Müller, M. and Schreyer, A., "The High Energy Materials Science Beamline (HEMS) at PETRA III," AIP Conference Proceedings 1234(1), 391-394 (2010).

[5] Momose, A., Kawamoto, S., Koyama, I., Hamaishi, Y., Takai, K. and Suzuki, Y., "Demonstration of X-Ray Talbot Interferometry," Jpn. J. Appl. Phys. 42(7B), L866 (2003).

[6] Snigirev, A., Snigireva, I., Kohn, V., Kuznetsov, S. and Schelokov, I., "On the possibilities of X-ray phase contrast microimaging by coherent high-energy synchrotron radiation," Review of Scientific Instruments 66(12), 5486-5492 (1995).

[7] Wilkins, S. W., Gureyev, T. E., Gao, D., Pogany, A. and Stevenson, A. W., "Phase-contrast imaging using polychromatic hard X-rays," Nature 384(6607), 335-338 (1996).

[8] Mittal, R., Morley, J., Dinopoulos, H., Drakoulakis, E. G., Vermani, E. and Giannoudis, P. V., "Use of bio-resorbable implants for stabilisation of distal radius fractures: the United Kingdom patients' perspective," Injury 36(2), 333-338 (2005).

[9] Kim, S. H., Oh, J. H., Lee, O.-S., Lee, H.-R. and Hargens, A. R., "Postoperative Imaging of Bioabsorbable Anchors in Rotator Cuff Repair," Am J Sports Med 42(3), 552-557 (2014).

[10] Virtanen, S., "Biodegradable Mg and Mg alloys: Corrosion and biocompatibility," Materials Science and Engineering: B 176(20), 1600-1608 (2011).

[11] Luthringer, B. J. C., Feyerabend, F. and Willumeit-Römer, R., "Magnesium-based implants: a mini-review," Magnesium Research 27(4), 142-154 (2014). 
[12] Sanchez, A. H. M., Luthringer, B. J. C., Feyerabend, F. and Willumeit, R., "Mg and Mg alloys: How comparable are in vitro and in vivo corrosion rates? A review," Acta Biomaterialia 13, 16-31 (2015).

[13] Barth, H. D., Launey, M. E., MacDowell, A. A., Ager, J. W. and Ritchie, R. O., "On the effect of X-ray irradiation on the deformation and fracture behavior of human cortical bone," Bone 46(6), 1475-1485 (2010).

[14] Barth, H. D., Zimmermann, E. A., Schaible, E., Tang, S. Y., Alliston, T. and Ritchie, R. O., "Characterization of the effects of x-ray irradiation on the hierarchical structure and mechanical properties of human cortical bone," Biomaterials 32(34), 8892-8904 (2011).

[15] Hubbell, J. H. and Seltzer, S. M., "Tables of X-Ray Mass Attenuation Coefficients and Mass Energy-Absorption Coefficients $1 \mathrm{keV}$ to $20 \mathrm{MeV}$ for Elements $\mathrm{Z}=1$ to 92 and 48 Additional Substances of Dosimetric Interest," http://physics.nist.gov/PhysRefData/XrayMassCoef/cover.html (1995).

[16] Chlebus, G., Meine, H., Moltz, J. H. and Schenk, A., "Neural Network-Based Automatic Liver Tumor Segmentation With Random Forest-Based Candidate Filtering," arXiv:1706.00842 [cs] (2017).

[17] Christ, P. F., Elshaer, M. E. A., Ettlinger, F., Tatavarty, S., Bickel, M., Bilic, P., Rempfler, M., Armbruster, M., Hofmann, F., D’Anastasi, M., Sommer, W. H., Ahmadi, S.-A. and Menze, B. H., "Automatic Liver and Lesion Segmentation in CT Using Cascaded Fully Convolutional Neural Networks and 3D Conditional Random Fields," arXiv:1610.02177 [cs] 9901, 415-423 (2016).

[18] Fauw, J. D., Ledsam, J. R., Romera-Paredes, B., Nikolov, S., Tomasev, N., Blackwell, S., Askham, H., Glorot, X., O’Donoghue, B., Visentin, D., Driessche, G. van den, Lakshminarayanan, B., Meyer, C., Mackinder, F., Bouton, S., Ayoub, K., Chopra, R., King, D., Karthikesalingam, A., et al., "Clinically applicable deep learning for diagnosis and referral in retinal disease," Nat Med 24(9), 1342-1350 (2018).

[19] Kayalibay, B., Jensen, G. and van der Smagt, P., "CNN-based Segmentation of Medical Imaging Data," arXiv:1701.03056 [cs] (2017).

[20] Ronneberger, O., Fischer, P. and Brox, T., "U-Net: Convolutional Networks for Biomedical Image Segmentation," Medical Image Computing and Computer-Assisted Intervention MICCAI 2015, N. Navab, J. Hornegger, W. M. Wells, and A. F. Frangi, Eds., 234-241, Springer International Publishing (2015).

[21] Kingma, D. P. and Ba, J., “Adam: A Method for Stochastic Optimization,” arXiv:1412.6980 [cs] (2014).

[22] Buslaev, A., Parinov, A., Khvedchenya, E., Iglovikov, V. I. and Kalinin, A. A., "Albumentations: fast and flexible image augmentations," arXiv:1809.06839 [cs] (2018).

[23] Chollet, F. and others., "Keras: The Python Deep Learning library," Astrophysics Source Code Library, ascl:1806.022 (2018).

[24] Horn, B. K. P. and Schunck, B. G., "Determining optical flow," Artificial Intelligence 17(1), 185-203 (1981).

[25] Brox, T., Bruhn, A., Papenberg, N. and Weickert, J., "High Accuracy Optical Flow Estimation Based on a Theory for Warping," Computer Vision - ECCV 2004, T. Pajdla and J. Matas, Eds., 25-36, Springer Berlin Heidelberg (2004). 
[26] Myagotin, A., Ershov, A., Helfen, L., Verdejo, R., Belyaev, A. and Baumbach, T., "Coalescence analysis for evolving foams via optical flow computation on projection image sequences," J Synchrotron Rad 19(4), 483-491 (2012).

[27] Ershov, A., "3D Optical flow using NVIDIA CUDA.," 22 March 2019, $<$ https://github.com/axruff/cuda-flow3d > (4 September 2019 ).

[28] Lautner, S., Zollfrank, C. and Fromm, J., "Microfibril Angle Distribution of Poplar Tension Wood," IAWA Journal 33(4), 431-439 (2012).

[29] Palenstijn, W. J., Batenburg, K. J. and Sijbers, J., "Performance improvements for iterative electron tomography reconstruction using graphics processing units (GPUs)," Journal of Structural Biology 176(2), 250-253 (2011).

[30] van Aarle, W., Palenstijn, W. J., De Beenhouwer, J., Altantzis, T., Bals, S., Batenburg, K. J. and Sijbers, J., "The ASTRA Toolbox: A platform for advanced algorithm development in electron tomography," Ultramicroscopy 157, 35-47 (2015).

[31] Moosmann, J., "Data reconstruction and analysis tools for tomography data.," 4 September 2019, <https://github.com/moosmann/matlab> (4 September 2019).

[32] Moosmann, J., Ershov, A., Weinhardt, V., Baumbach, T., Prasad, M. S., LaBonne, C., Xiao, X., Kashef, J. and Hofmann, R., "Time-lapse X-ray phase-contrast microtomography for in vivo imaging and analysis of morphogenesis," Nature Protocols 9(2), 294-304 (2014).

[33] Dean, M. N., Mull, C. G., Gorb, S. N. and Summers, A. P., "Ontogeny of the tessellated skeleton: insight from the skeletal growth of the round stingray Urobatis halleri," Journal of Anatomy 215(3), 227-239 (2009).

[34] Dean, M. N., Seidel, R., Knötel, D., Lyons, K., Baum, D., Weaver, J. C. and Fratzl, P., “To build a shark: 3D tiling laws of tessellated cartilage" (2016).

[35] Moosmann, J., Ershov, A., Altapova, V., Baumbach, T., Prasad, M. S., LaBonne, C., Xiao, X., Kashef, J. and Hofmann, R., "X-ray phase-contrast in vivo microtomography probes new aspects of Xenopus gastrulation," Nature 497(7449), 374-377 (2013).

[36] Hipp, A., Moosmann, J., Herzen, J., Hammel, J. U., Schreyer, A. and Beckmann, F., "Highresolution grating interferometer for phase-contrast imaging at PETRA III," Developments in X-Ray Tomography XI 10391, 1039108, International Society for Optics and Photonics (2017).

[37] Hipp, A., Beckmann, F., Lytaev, P., Greving, I., Lottermoser, L., Dose, T., Kirchhof, R., Burmester, H., Schreyer, A. and Herzen, J., "Grating-based x-ray phase-contrast imaging at PETRA III," Developments in X-Ray Tomography IX 9212, 921206, International Society for Optics and Photonics (2014). 